\begin{tabular}{|c|c|c|c|}
\hline Victoria Poplin, MD & Brent Harbaugh, D0, MA & Matthias Salathe, MD & Nathan C. Bahr, MD, MA \\
\hline Division of Infectious Diseases, & Department of Pathology, & Division of Pulmonary, Critical Care, & Division of Infectious Diseases, \\
\hline epartment of Internal Medicine, & University of Kansas, & and Sleep Medicine, Department & Department of Internal Medicine, \\
\hline $\begin{array}{l}\text { University of Kansas, } \\
\text { Kansas City, KS }\end{array}$ & Kansas City, KS & $\begin{array}{l}\text { of Internal Medicine, University of } \\
\text { Kansas, Kansas City, KS }\end{array}$ & ra \\
\hline
\end{tabular}

\title{
Miliary tuberculosis in a patient with end-stage liver disease
}

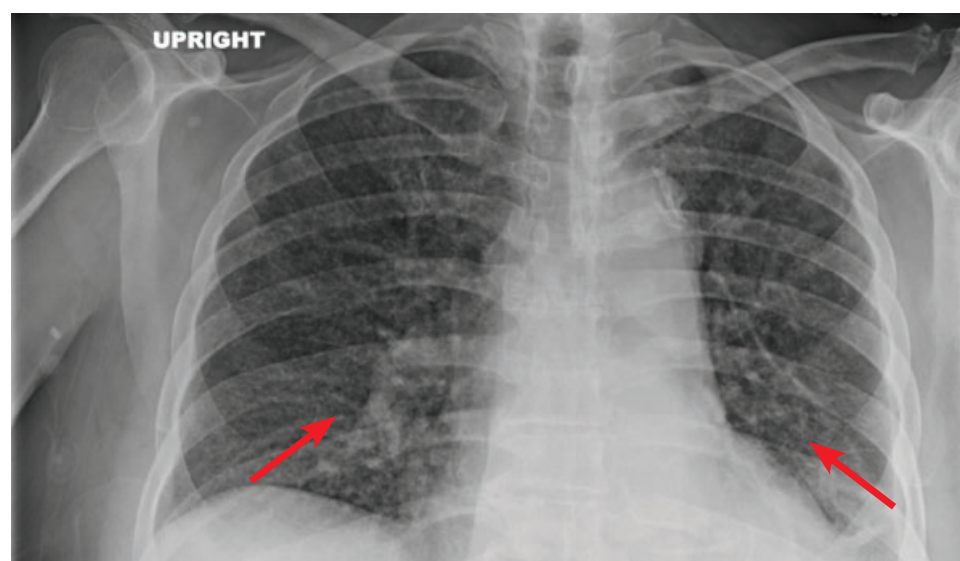

Figure 1. Chest radiography showed patchy left basilar consolidation with subtle, diffuse interstitial opacities (arrows) throughout both lungs.

\footnotetext{
52-YeAR-Old Mexican American man A with end-stage liver disease due to alcohol abuse was admitted to the hospital because of altered mental status.

One month earlier, he had been hospitalized for culture-negative spontaneous bacterial peritonitis, at which time his liver disease was diagnosed on the basis of his history of heavy alcohol abuse, results of imaging and laboratory testing, and clinical findings. Liver biopsy was not performed at that time.

During that hospitalization, he had recurrent fevers that were attributed to bacterial pneumonia based on a chest radiograph that showed patchy left basilar consolidation with subtle, diffuse interstitial opacities throughout both lungs (Figure 1). This was treated with ceftriaxone.

He underwent additional testing that included viral hepatitis serologies, testing for human immunodeficiency virus (HIV), and

bacterial blood cultures; all were negative. Also negative were bacterial culture of the peritoneal fluid, acid-fast bacillus staining of the peritoneal fluid, and fungal staining of the peritoneal fluid; acid-fast bacillus culture and fungal culture of the peritoneal fluid were pending at discharge. No other workup for respiratory infection was done at that time.

\section{INITIAL WORKUP}

At the time of the current admission, the patient was confused and unable to communicate. He was a known smoker, but whether he used e-cigarettes was not known.

Results of initial laboratory testing were as follows:

- Platelet count $54 \times 10^{9} / \mathrm{L}$ (reference range 150-400), dropping rapidly to less than 10 $\times 10^{9} / \mathrm{L}$

- Hemoglobin 11.7 g/dL (13.5-16.5)

- Sodium $119 \mathrm{mmol} / \mathrm{L}(137-147)$

- Creatinine $1.76 \mathrm{mg} / \mathrm{dL}(0.4-1.24)$.

Computed tomography (CT) of the chest without contrast revealed tiny nodular opacities in all lobes (Figure 2) and an irregular lytic lesion in the T9 vertebral body (Figure $3)$.

Three sputum acid-fast bacilli smears were negative.

The differential diagnosis was broad and included disseminated histoplasmosis, coccidioidomycosis, candidal infection, tuberculosis, cryptococcosis, cancer metastasis (particularly hepatocellular carcinoma or lung cancer, given his risk factors), sarcoidosis, silicosis, and hypersensitivity pneumonitis. Disseminated fungal or mycobacterial disease was believed to be more likely, based on the duration and progression of symptoms despite antibiotics (cef- 


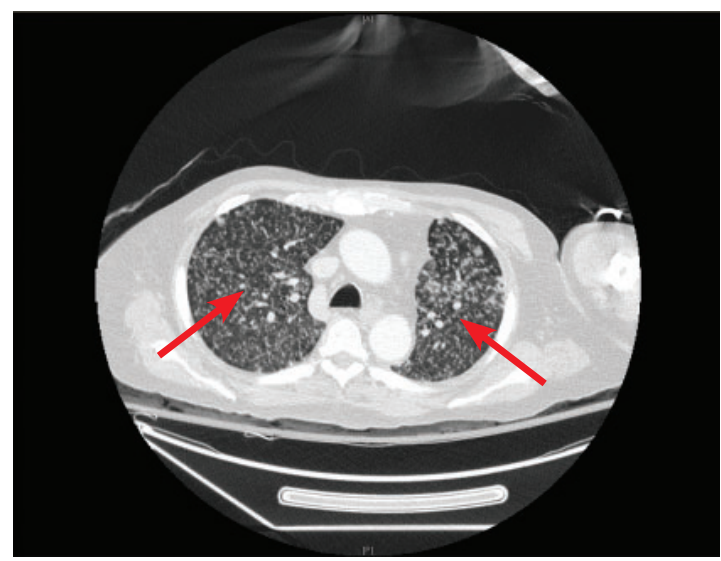

Figure 2. Computed tomography without contrast revealed tiny nodular opacities (arrows) in all lobes.

triaxone for 7 days in the prior hospitalization, and levofloxacin started during this admission), the CT findings, and the recent negative peritoneal fluid culture. Hepatic encephalopathy was an initial consideration; however, his mentation did not improve with lactulose.

Sputum staining was negative for bacteria, fungi, and acid-fast bacilli.

Empiric treatment for tuberculosis was started with rifampin, ethambutol, levofloxacin, and 3-times-weekly amikacin. (Amikacin and levofloxacin were substituted for isoniazid and pyrazinamide due to his liver disease.) The empiric treatment was based on CT findings and a clinical scenario consistent with miliary tuberculosis in a patient with underlying liver disease who came from a country in which tuberculosis is common.

More tests for infections were negative: a respiratory viral panel, urine Histoplasma antibodies and antigen, Legionella antigen, HIV screening, bacterial and fungal sputum cultures, cryptococcal antigen, Coccidioides antibody and antigen, Blastomyces antigen, Aspergillus antigen, and (1-3)-beta-d-glucan.

He underwent bronchoalveolar lavage; the fluid had 360 cells $/ \mu \mathrm{L}$, with $56 \%$ monocytes, 23\% lymphocytes, and 20\% neutrophils. Pneumocystis polymerase chain reaction (PCR) testing, cytology, herpes simplex PCR, and bacterial culture of the fluid were negative.

Throughout the hospital stay, the patient's severe thrombocytopenia failed to improve despite multiple transfusions and intravenous

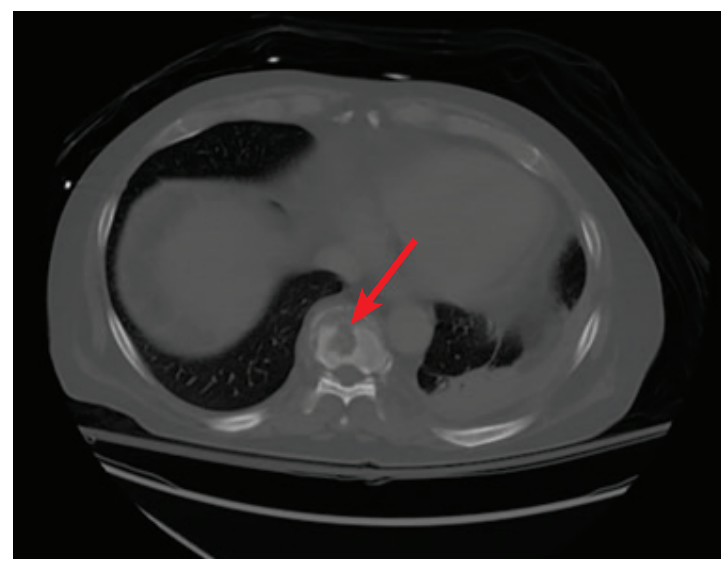

Figure 3. Computed tomography without contrast revealed an irregular lytic lesion in the T9 vertebral body (arrow).

immunoglobulin. His platelet count eventually stabilized at 20 to $30 \times 10^{9} / \mathrm{L}$.

\section{HEMATOLOGIC EVALUATION}

Hematologic conditions in the differential diagnosis in this patient included disseminated intravascular coagulation, idiopathic thrombocytopenic purpura, thrombotic thrombocytopenic purpura, immune-mediated destruction, splenic sequestration, and bone marrow infiltration from infection.

A hematologic workup revealed normal haptoglobin, mildly elevated lactate dehydrogenase at $232 \mathrm{U} / \mathrm{L}$ (reference range 135-225), total bilirubin $1.3 \mathrm{mg} / \mathrm{dL}(0.2-1.3)$, and direct bilirubin $0.3 \mathrm{mg} / \mathrm{dL}(<0.2)$. Serum protein electrophoresis showed hypoalbuminemia and increased kappa and lambda free light chains with a normal ratio. Heparin-induced platelet antibody and platelet antibody testing were negative. Fibrinogen was reduced at $197 \mathrm{mg} /$ $\mathrm{dL}$, the international normalized ratio was elevated at 1.4 , and the activated partial thromboplastin time was normal.

The initial immature platelet fraction was inappropriately normal at $6.0 \%$, but was elevated at $32.8 \%$ on repeat testing 10 days later.

Peripheral smear showed normocytic anemia with anisocytosis, absolute lymphocytopenia, and marked thrombocytopenia with normal platelet morphology. No schistocytes or platelet clumping were seen.

He underwent bone marrow biopsy. The marrow was mildly hypercellular (50\%) with
The severe

thrombocytopenia failed to improve despite multiple transfusions and IV immunoglobulin 
mild pancellular hyperplasia and multiple granulomas, a few of which were necrotizing. Acid-fast bacillus staining was negative. Megakaryocytes were increased, with normal morphology.

\section{THROMBOCYTOPENIA WITH MULTIPLE CONTRIBUTING FACTORS}

Based on the evaluation, the patient's thrombocytopenia was multifactorial, due to causes that included underlying cirrhosis, likely infiltration of Mycobacterium tuberculosis, and a possible immune-mediated component, which was considered because of the brisk fall in platelets after transfusion early in the hospital course and improvement in the rate of decline later in the course (after tuberculosis therapy, possibly due to decreased tuberculosis antigen load).

The diagnosis of miliary tuberculosis with pulmonary and peritoneal involvement was confirmed when a sputum culture grew $\mathrm{M} t u$ berculosis, as did a peritoneal fluid culture from the previous hospitalization.

Bone, bone marrow, and meningeal in-

Clinical

findings

of miliary

tuberculosis are nonspecific and may include fever, weight loss, anorexia, tachycardia, and night sweats for more than 6 weeks gressive multiorgan dysfunction that led to a fatal arrhythmia.

\section{FEATURES OF MILIARY TUBERCULOSIS}

Miliary tuberculosis is a rare form of tuberculosis, accounting for up to $2 \%$ of cases. The mortality rate is up to $33 \%$, in large part due to delay in diagnosis, which often happens in areas of low prevalence such as the United States. ${ }^{1,2}$ A risk factor in this case was that the patient was born in Mexico, and though he had lived in the United States for more than 10 years, he occasionally traveled to Mexico.

The differential diagnosis for this patient's altered mental status and progressive pulmonary imaging abnormalities was broad. However, the history of culture-negative spontaneous bacterial peritonitis and progressive abnormalities on CT for more than 1 month despite treatment of bacterial pneumonia made disseminated fungal or mycobacterial disease more likely, and as noted, the workup rapidly pointed to tuberculosis during the second admission, and all tests for fungal infectious were negative.

\section{Clinical findings}

Clinical findings of miliary tuberculosis are nonspecific and may include fever, weight loss, anorexia, tachycardia, and night sweats for more than 6 weeks. There is no standardized approach to diagnosis, ${ }^{2}$ but clinical clues that can be useful include symptoms consistent with tuberculosis, a classic miliary pattern or bilateral diffuse reticulonodular lung lesions on a background of miliary shadows on chest imaging, and microbiologic, histopathologic, or molecular evidence. ${ }^{2}$

\section{Approaches to diagnosis}

Radiographically, the classic miliary pattern is a widespread collection of uniform, discrete pulmonary opacities $2 \mathrm{~mm}$ or less in diameter. CT may show associated reticulation. ${ }^{2}$ The miliary pattern may be seen on CT even when a chest radiograph is normal. ${ }^{2}$

Acid-fast bacillus smears, mycobacterial culture, or nucleic acid amplification testing of any involved tissue or fluid (eg, sputum, cerebrospinal fluid, ascites, bone marrow, lymph tissue) can be used to confirm the presence of M tuberculosis. ${ }^{2-4}$ The diagnostic yield can vary 
drastically depending on the test and the tissue being tested. ${ }^{4}$ Sharma and Mohan ${ }^{4}$ combined data from several studies and calculated that culture and acid-fast bacillus smear had a sensitivity of $41 \%$ in sputum, $21 \%$ in cerebrospinal fluid, and $33 \%$ in urine.

Although combining data from different cohorts has limitations, the general principle that the diagnostic test performance varies by specimen type (and degree of bacillary prevalence) is accurate. For example, in cerebrospinal fluid, the sensitivity of acid-fast bacillus smear is $10 \%$ to $15 \%$ (though it is rapid and cheap), while culture is slow (4-6 weeks) and has $50 \%$ to $60 \%$ sensitivity, similar to the more rapid approved PCR assays (2-48 hours). ${ }^{5}$

Abnormalities on laboratory testing can include pancytopenia, hyponatremia (especially with meningeal involvement), hypercalcemia, and disseminated intravascular coagulation, though none of these are specific to miliary tuberculosis. $2,4,6$

Liver biopsy and lymph node biopsy, while invasive, tend to have higher yields. ${ }^{4}$ Patients with cirrhosis are likely susceptible to tuberculosis infection due to immune dysfunction. ${ }^{?}$ Extrapulmonary tuberculosis, including peritoneal and miliary types, is more common in patients with cirrhosis. ${ }^{8-10}$ Both miliary tuberculosis and underlying cirrhosis have been shown to be independent predictors of death in patients with tuberculosis. ${ }^{7,11}$

\section{REFERENCES}

1. Sharma SK, Mohan A, Sharma A. Challenges in the diagnosis \& treatment of miliary tuberculosis. Indian J Med Res 2012; 135(5):703-730. pmid:22771605

2. Sharma SK, Mohan A, Sharma A. Miliary tuberculosis: a new look at an old foe. J Clin Tuberc Other Mycobact Dis 2016; 3:13-27. doi:10.1016/j.jctube.2016.03.003

3. Lewinsohn DM, Leonard MK, LoBue PA, et al. Official American Thoracic Society/Infectious Diseases Society of America/Centers for Disease Control and Prevention clinical practice guidelines: diagnosis of tuberculosis in adults and children. Clin Infect Dis 2017 64(2):111-115. doi:10.1093/cid/ciw778

4. Sharma SK, Mohan A. Miliary tuberculosis. Microbiol Spectr 2017; 5(2). doi:10.1128/microbiolspec.TNMI7-0013-2016

5. Bahr NC, Meintjes G, Boulware DR. Inadequate diagnostics: the case to move beyond the bacilli for detection of meningitis due to Mycobacterium tuberculosis. J Med Microbiol 2019; 68(5):755-760. doi:10.1099/jmm.0.000975

6. Nahid P, Dorman SE, Alipanah N, et al. Official American Thoracic Society/Centers for Disease Control and Prevention/Infectious Diseases Society of America clinical practice guidelines: treatment of drug-susceptible tuberculosis. Clin Infect Dis 2016; 63(7):e147-e195. doi:10.1093/cid/ciw376

\section{Treatment}

Tuberculosis treatment-related hepatotoxicity is more common in patients with underlying cirrhosis. ${ }^{10}$ All of these factors were working against the patient in this case.

If the tuberculosis is drug-susceptible, treatment includes isoniazid, rifampin, ethambutol, and pyrazinamide for the first 2 months, followed by 4 months of isoniazid and rifampin (extended to a total of 9-12 months with bone or meningeal involvement).,3,6 If meningeal involvement is suspected, a higher dosage of rifampin $(30 \mathrm{mg} / \mathrm{kg} /$ day $)$ should be considered to improve central nervous system penetration, and adjunctive corticosteroids are recommended..$^{12}$

In our patient, treatment was complicated by liver disease. There are currently no standardized treatments for tuberculosis in patients with severe liver disease. A regimen recommended by the Infectious Diseases Society of America and American Thoracic Society is rifampin and ethambutol for 12 months, with a fluoroquinolone for the first 2 months. ${ }^{3}$ Any case of tuberculosis in a patient with underlying liver disease should prompt expert consultation.

In our patient, we used high-dose rifampin, ethambutol, levofloxacin, and, briefly, amikacin. Unfortunately, we were not able to cure him, and he died.

7. Hung TH, Lay CJ, Tseng CW, Tsai CC, Tsai CC. The effect of tuberculosis on the mortality of cirrhotic patients: a population-based 3-year follow-up study. Medicine (Baltimore) 2014; 93(28):e295. doi:10.1097/MD.0000000000000295

8. Sharma P, Tyagi P, Singla V, Bansal N, Kumar A, Arora A. Clinical and biochemical profile of tuberculosis in patients with liver cirrhosis. J Clin Exp Hepatol 2015; 5(1):8-13. doi:10.1016/j.jceh.2015.01.003

9. Aguado JM, Pons F, Casafont F, San Miguel G, Valle R. Tuberculous peritonitis: a study comparing cirrhotic and noncirrhotic patients. J Clin Gastroenterol 1990; 12(5):550-554. pmid:2146314

10. Cho YJ, Lee SM, Yoo CG, et al. Clinical characteristics of tuberculosis in patients with liver cirrhosis. Respirology 2007; 12(3):401-405. doi:10.1111/j.1440-1843.2007.01069.x

11. Lin CH, Lin CJ, Kuo YW, et al. Tuberculosis mortality: patient characteristics and causes. BMC Infect Dis 2014; 14:5. doi:10.1186/1471-2334-14-5

12. Dian S, Yunivita V, Ganiem AR, et al. Double-blind, randomized, placebo-controlled phase II dose-finding study to evaluate high-dose rifampin for tuberculous meningitis. Antimicrob Agents Chemother 2018; 62(12):e01014-e01018. doi:10.1128/AAC.01014-18

Address: Nathan C. Bahr, MD, MA, Department of Internal Medicine,

Division of Infectious Diseases. University of Kansas Mailstop 1028, 3901

Rainbow Boulevard, Kansas City, KS 66160; nbahr@kumc.edu 